\title{
Influencia de la conducta antisocial en el consumo de drogas ilegales en población adolescente
}

\author{
Muñoz-Rivas, M.J.*; Graña Gómez, J.L.**; Peña Fernández, M.e.***; Andreu Rodríguez, J.M.*** \\ * Profesora Asociada. Departamento de Psicología Clínica. Facultad de Psicología. Universidad Complutense de Madrid. \\ * Profesor Titular. Departamento de Psicología Clínica. Facultad de Psicología. Universidad Complutense de Madrid. \\ *** Becarios de Investigación. Departamento de Psicología Clínica. Facultad de Psicología. Universidad Complutense de Madrid. \\ Enviar correspondencia: \\ José Luis Graña Gómez. Departamento de Psicología Clínica. Facultad de Psicología de la Universidad Complutense de Madrid. Campus de Somosaguas. \\ 28223 Madrid. Tel. 9139431 28. E-mail: jlgrana@psi.ucm.es
}

\section{RESUMEN}

Son muchos los estudios que han considerado que en la fase de inicio en el uso de sustancias adictivas, que suele darse de forma generalizada en la adolescencia, influyen varios factores psicológicos y de personalidad entre los que destacan la búsqueda de sensaciones y la impulsividad, además de la conducta antisocial, objeto del presente estudio. En este caso, el objetivo principal fue determinar la influencia diferencial de la conducta antisocial en el consumo de drogas ilegales (cannabis, derivados morfínicos, estimulantes, drogas sintéticas y cocaína) utilizando una muestra de 1570 adolescentes de la Comunidad de Madrid a través de la "Escala de Conducta Antisocial" (Allsopp y Feldman, 1976) así como de un cuestionario elaborado expresamente que estimó el consumo de las distintas sustancias de comercio ilegal. Los resultados obtenidos señalan una estrecha relación entre un mayor y más frecuente uso de estas sustancias y una mayor presencia de conductas contra las normas. A diferencia de los varones, las mujeres que exhiben un comportamiento más antisocial realizan un consumo de estimulantes y drogas sintéticas significativamente superior que aquellas en las que este tipo de conductas es menos frecuente.

Palabras Clave: Drogas ilegales, Conducta antisocial, Adolescentes, factores de riesgo.

\section{ABSTRACT}

Many studies have considered that the earlier stages of addictive substance use, which normally appear in adolescence, are influenced by various psychological and personality aspects, among which the most relevant are the seeking of new sensations and impulsiveness but also antisocial behaviour, which is the subject of the present study. In this case, the main objective was to determine the differential influence of antisocial behaviour in the consumption of illegal drugs (cannabis, morphine-derived substances, stimulants, synthetic drugs, cocaine) in a sample of 1570 adolescents from the Madrid Region utilising the Antisocial Behaviour Scale (Allsopp and Feldman, 1976) and an ad hoc questionnaire designed for this study to estimate the use of the different substances. The results obtained show a close connection between a higher and more frequent use of the substances stated above and a higher presence of behaviours against social norms. Unlike the males, the females, who show a more antisocial behaviour have a significantly higher consumption of stimulants and synthetic drugs than those who show this kind of conduct less frequently.

Key words: drugs, antisocial behaviour, adolescents, risk factors.

\section{INTRODUCCIÓN}

E estudio de las variables de personalidad entendidas como factores de riesgo o de vulnerabilidad para el uso/abuso de drogas ha sido uno de los campos menos desarrollados desde un punto de vista genérico. Sólo determinados aspectos, como la búsqueda de sensaciones y la presencia de problemas de conducta tempranos (personalidad antisocial) que a continuación comentaremos, han tenido un mayor soporte e interés experimental (véase tabla 1).
En este ámbito, estudios centrados en el análisis de rasgos de personalidad en la infancia y adolescencia que pudieran estar implicados en el posterior consumo de sustancias tanto legales como ilegales, han señalado repetidamente la relevancia de determinadas variables psicológicas como son un pobre control de las emociones, la inconvencionalidad y la introversión social (Brook, et al.,1989) y la timidez, la agresividad y la personalidad depresiva (Kellam, et al., 1983). Los trabajos en este área parten del supuesto de que cuando aparecen en una edad temprana conductas 
Tabla 1. Rasgos de personalidad como factores de riesgo en el consumo de drogas

\begin{tabular}{|c|c|c|}
\hline FACTOR DE RIESGO & ESTUDIOS ETIOLÓGICOS & EVIDENCIA EMPÍRICA \\
\hline \multirow[t]{3}{*}{$\begin{array}{l}\text { Características de la } \\
\text { personalidad }\end{array}$} & Kellam et al., 1983. & $\begin{array}{l}\text { Rasgos como la timidez, la agresividad y la personalidad } \\
\text { depresiva en la infancia llegan a predecir el uso posterior de } \\
\text { drogas en la adolescencia. }\end{array}$ \\
\hline & Brook et al., 1989. & $\begin{array}{l}\text { Variables como un pobre control de las emociones, la inconve- } \\
\text { nionalidad y la introversión social se asocian estrechamente al } \\
\text { inicio y la escalada en el consumo de drogas. }\end{array}$ \\
\hline & $\begin{array}{l}\text { Aronson y Gilbert, 1963; Nylander, 1960; } \\
\text { Winokur et al., 1970; Herjanic et al., 1977; } \\
\text { Schuckit, 1982, 1983; McKenna y Pickens, } \\
\text { 1983; Knowles y Schroeder, } 1990 .\end{array}$ & $\begin{array}{l}\text { En hijos de alcohólicos, se da una mayor presencia de rasgos } \\
\text { antisociales, de una personalidad de tipo pasivo-agresiva, de } \\
\text { rasgos de introversión, desadaptación social e impulsividad y } \\
\text { otros signos de incapacidad manifiesta para desarrollar y man- } \\
\text { tener relaciones gratificantes. }\end{array}$ \\
\hline \multirow[t]{3}{*}{$\begin{array}{l}\text { (a) Búsqueda de } \\
\text { sensaciones }\end{array}$} & $\begin{array}{l}\text { Zuckerman, 1979, 1980, 1987; Bates et al., } \\
\text { 1986; Huba et al., 1986; Horovath y } \\
\text { Zuckerman, 1993. }\end{array}$ & $\begin{array}{l}\text { Estrecha relación enre la búsqueda de sensaciones y determi- } \\
\text { nadas conductas como el uso del alcohol y otras drogas ilega- } \\
\text { les y la criminalidad. }\end{array}$ \\
\hline & $\begin{array}{l}\text { Pedersen et al., 1988, 1989; Teichman et al., } \\
\text { 1989; Levenson, 1990; Joe y Simpson, } 1991 .\end{array}$ & $\begin{array}{l}\text { Fuertes correlaciones entre esta variable de personalidad y el } \\
\text { consumo de sustancias señalando un poder predictivo similar } \\
\text { o superior al de otros factores como la excitabilidad emocio- } \\
\text { nal, la depresión y la influencia de padres y amigos. }\end{array}$ \\
\hline & $\begin{array}{l}\text { Cárdenas y Moreno-Jiménez, 1989; } \\
\text { Del Barrio y Alonso, } 1994 .\end{array}$ & $\begin{array}{l}\text { En población española, se confirma la asociación entre la bús- } \\
\text { queda de sensaciones y el consumo de drogas legales (taba- } \\
\text { co y alcohol). }\end{array}$ \\
\hline \multirow[t]{6}{*}{$\begin{array}{l}\text { (b) Personalidad } \\
\text { antisocial }\end{array}$} & Robins, 1978. & $\begin{array}{l}\text { La mayor variedad y frecuencia de aparición de conductas anti- } \\
\text { sociales en la infancia predice,en la edad adulta, la persisten- } \\
\text { cia de conductas asociales más peligrosas, incluido el } \\
\text { consumo de drogas. }\end{array}$ \\
\hline & $\begin{array}{l}\text { Nylander, 1979; Kellam y Brown, 1982; } \\
\text { Spivack, 1983; Lewis et al., } 1985 .\end{array}$ & $\begin{array}{l}\text { La agresividad en niños de 5-7 años, predice el uso frecuente } \\
\text { de drogas y la delincuencia en la adolescencia y en la edad } \\
\text { adulta. }\end{array}$ \\
\hline & Loeber y Dishion, 1983. & $\begin{array}{l}\text { Sólo el } 30-40 \% \text { de los chicos con problemas de conducta } \\
\text { agresiva, los mantienen de } 4 \text { a } 9 \text { años más tarde. }\end{array}$ \\
\hline & $\begin{array}{l}\text { McCord, 1981; Kandel, 1982; Barnes y Welte, } \\
\text { 1986; Loeber, } 1988 .\end{array}$ & $\begin{array}{l}\text { Si la conducta agresiva presente en la infancia persiste en la } \\
\text { adolescencia, es un potente predictor del alcoholismo, del } \\
\text { abuso de drogas y de la conducta agresiva en años posterio- } \\
\text { res. }\end{array}$ \\
\hline & Gittelman et al., 1985. & $\begin{array}{l}\text { La hiperactividad en niños, especialmente si va acompañada } \\
\text { por problemas de conducta, incrementa el riesgo de abuso de } \\
\text { sustancias en la adolescencia. }\end{array}$ \\
\hline & Brook et al., 1990. & $\begin{array}{l}\text { Los niños irritables, fácilmente distraibles, con frecuentes } \\
\text { rabietas y peleas con los hermanos y con conductas predelin- } \\
\text { cuentes tienen mayor probabilidad de consumir alcohol y otras } \\
\text { drogas en la adolescencia. }\end{array}$ \\
\hline
\end{tabular}

antisociales frecuentes y variadas, lo más probable es que persistan en la edad adulta (Robins, 1978) y que favorezcan el desarrollo posterior de otro tipo de comportamientos de riesgo más peligrosos como es el uso/abuso de drogas. En esta línea, Brook et al. (1990), encontraron que los niños irritables, fácilmente distraibles, con rabietas y peleas frecuentes con sus hermanos y especialmente implicados en el desa- 
rrollo de conductas predelincuentes (ej., pequeños hurtos, mentiras, engaños) eran más proclives al uso de drogas en la adolescencia que aquellos que no exhibían tales conductas.

Más concretamente, la conducta agresiva en niños parece ser una señal de la conducta antisocial posterior. Se ha encontrado que la agresividad en niños entre los 5 y los 7 años puede ser un potente predictor de la conducta antisocial posterior, en la que se incluyen tanto la frecuencia de uso de drogas en la adolescencia (Kellam y Brown, 1982) y los problemas de dependencia por abuso de sustancias psicoactivas en la edad adulta (Nylander, 1979; Lewis, et al., 1985) como la delincuencia en la etapa adolescente (Spivack, 1983; Loeber, 1988). De cualquier modo, es necesario señalar que la agresividad temprana no va invariablemente seguida de una conducta antisocial seria en la etapa adulta. Según los estudios, sólo alrededor del 30-40\% de los niños con conductas desadaptadas y agresivas, continúa exhibiéndolas entre 4 y 9 años más tarde (Loeber y Dishion, 1983). Así, de las muestras estudiadas, pocos jóvenes desarrollaron conductas agresivas físicas al final de la infancia o la adolescencia si no habían desarrollado tales conductas en una temprana infancia y, la mayoría de ellos, superaron las conductas agresivas iniciales. De cualquier modo, los datos parecen señalar que si la conducta agresiva continúa hasta el comienzo de la adolescencia (13 años), puede ser considerada como un potente predictor del abuso de sustancias como el alcoholismo y de la conducta agresiva en los años posteriores (McCord, 1981; Loeber, 1988). Además, el abuso de drogas parece hacerse más probable si la conducta antisocial persiste y se vuelve más variada en la adolescencia temprana incluyendo peleas y mal comportamiento en el ámbito escolar (Kandel, 1982; Barnes y Welte, 1986).

Finalmente, subrayar que también variables como la hiperactividad y los desórdenes por déficit de atención parecen incrementar el riesgo de delinquir cuando se combinan con problemas de conducta incluyendo la agresión en todas sus formas. En este sentido, Gittelman, et al., (1985), encontraron una alta prevalencia de trastornos por abuso de sustancias y emisión de conductas delictivas (ej, robos, asaltos) en la adolescencia tardía entre sujetos diagnosticados de hiperactividad en la infancia. A pesar de la importancia del resultado, este descubrimiento que propone que la hiperactividad $\sin$ ir acompañada de problemas de conducta puede predecir por sí sola un riesgo aumentado para el abuso de sustancias, no ha sido replicado. Sin embargo, sugiere la importancia de realizar investigaciones sobre la relación entre los trastornos por déficit de atención, los problemas de conducta y el abuso de sustancias.

Teniendo en cuenta los aspectos comentados, el presente estudio se propuso con el objetivo de eva- luar, en primer lugar, la posible de influencia de conductas antisociales en el uso/abuso de drogas ilegales en una muestra de población adolescente representativa de la Comunidad Autónoma de Madrid. Y, en segundo lugar, en el caso de existir una relación significativa entre ambos factores, poner de manifiesto la presencia de posibles diferencias en función del comportamiento antisocial y el sexo de los jóvenes consumidores en cada una de las sustancias consumidas.

\section{MÉTODO}

\section{Sujetos}

Para el presente estudio se contó con la participación de un total de 1742 adolescentes pertenecientes bien a diversos cursos de la Educación Secundaria o bien a primero y segundo de Formación Profesional. El total de los participantes fue extraído de seis institutos de Madrid, representativos de distintas zonas sociodemográficas de la Comunidad con el objetivo de que estuvieran representados todos los niveles socioeconómicos y culturales.

Los centros escolares fueron seleccionados al azar de un total de diez que fueron propuestos inicialmente como posibles participantes por tener características similares y contar con la colaboración de los respectivos Departamentos de Orientación. Dentro de cada uno de los centros de enseñanza, el muestreo se realizó por conglomerados tomando el aula como unidad muestral. Se eligió al azar las aulas participantes de cada curso de Educación Secundaria y Formación Profesional tomando en consideración la disponibilidad de los profesores y de los alumnos dependiendo del programa de la asignatura.

Todos los sujetos participaron de forma voluntaria en el desarrollo de la prueba y eran conocedores de que ésta formaba parte de una investigación realizada desde la Facultad de Psicología de la Universidad Complutense de Madrid.

\section{Instrumentos}

Teniendo en cuenta los criterios generales de la investigación se creó un instrumento de evaluación que se adecuase a los objetivos perseguidos. La idea fundamental fue elaborar un formato que incluyera cuestiones referidas tanto al consumo de sustancias como al análisis de la presencia de determinados factores psicológicos y de personalidad que, según la literatura pueden explicar el inicio del mismo. Así pues, se recogieron, en primer lugar, datos relativos al consumo de distintas drogas ilegales (cannabis, derivados morfínicos, estimulantes, drogas de síntesis y 
cocaína) a través de diversos parámetros temporales como el uso de cada una de ellas "en los últimos treinta días" (consumo frecuente), donde el sujeto debía indicar la frecuencia con que lo hacía en un rango de respuesta tipo Likert que abarcaba desde "nada", "menos de una vez a la semana", "una vez por semana", "de dos a seis veces por semana", "una vez diariamente" y "dos o más veces diariamente". Además, debía anotar la edad en la que comenzó el consumo en el caso de haber probado cualquiera de las sustancias indicadas. En segundo lugar, se administró la Escala de conducta antisocial (ASB)(Silva, Martorell y Clemente, 1986), versión adaptada de la escala ASB desarrollada por Allsop y Feldman (1976). Consta de un total de 46 ítems y tiene por objetivo detectar aquellas conductas desviadas de las normas de comportamiento social típicas de la edad así como algunas conductas predelictivas en niños y adolescentes. Todos los ítems hacen referencia a conductas autoobservables en las que el sujeto no debe hacer ninguna inferencia relacionada con su estado interno. Para cada uno de ellos, la alternativa de respuesta es SI o NO. El sujeto debe responder marcando con un aspa sobre la respuesta que mejor se adecue a su caso. La escala ofrece una puntuación total que supone la suma de todas las respuestas afirmativas otorgándoles el valor 1 a cada una de ellas.

Para el estudio de la fiabilidad del instrumento, se tuvo en cuenta tanto la consistencia interna del mismo aplicando el coeficiente alfa de Cronbach obteniéndose un índice de 0.92, como la estabilidad temporal que ofreció un índice de 67 para un intervalo temporal de 4 meses y de 0.62 para el de un año.

\section{Procedimiento}

El primer paso fue seleccionar a priori un total de diez institutos de la Comunidad de Madrid que cumplieran una serie de requisitos mínimos: (a) tener un número de alumnos no inferior a 1000; (b) estar ubicados en distintas zonas de la Comunidad para poder abarcar distintos niveles socioculturales; (c) disponer de un Departamento de Orientación a través del cual organizar la evaluación de los sujetos y (d) mostrar su interés en el desarrollo de este tipo de investigaciones sobre el consumo de sustancias en un primer contacto telefónico. De todos ellos, fueron seleccionados al azar los seis centros que finalmente participaron en el estudio.

La aplicación del instrumento de evaluación se llevó a cabo de forma colectiva en cada una de las aulas representativas de cada uno de los cursos evaluados y los participantes dispusieron de un total de cincuenta minutos para su cumplimentación. La aplicación de la totalidad de las pruebas fue realizada por profesionales entrenados en ausencia de los profeso- res para evitar que el alumnado se sintiera influido a la hora de contestar al respecto de sus conductas de consumo.

\section{RESULTADOS}

Una vez eliminados aquellos cuestionarios con datos defectuosos y aquellos contestados aleatoriamente por los sujetos, se contó con una muestra definitiva de 1570 sujetos de ambos sexos (54,4\% hombres y $45,6 \%$ mujeres) con edades comprendidas entre los 14 y los 18 años (edad media=15,83).

Se analizaron las drogas ilegales conjunta y aisladamente consideradas a través de un MANOVA (Análisis Multivariante de la Varianza), tomando como variables dependientes la frecuencia de consumo, medida en una escala tipo Likert, del cannabis, los derivados morfínicos, los estimulantes, las drogas sintéticas y la cocaína. Como variables independientes se utilizaron el sexo y el factor psicológico estimado agrupando a los sujetos en función de los centiles 30 y 70, conformándose así dos grupos, altos y bajos en conducta antisocial. Por otra parte, la edad se utilizó como covariable para controlar el posible influjo que pudiera ejercer sobre las puntuaciones de las variables dependientes.

\section{Efecto de la conducta antisocial sobre el consumo de drogas ilegales}

Los análisis multivariantes realizados sobre el conjunto de sustancias analizadas mostraron un efecto significativo de la conducta antisocial $[F(5,900)=$ $25,90, p<0,001]$ y del sexo $[F(5,900)=5,51, p<0,001]$, así como efectos de interacción entre ambos factores $[F(5,900)=2,52, p<0,05]$.

Tomando cada una de las sustancias aisladamente consideradas, los análisis univariantes realizados (ANOVA) señalaron un efecto significativo de la conducta antisocial en cada uno de los casos (Gráfico 1). Así pues, los adolescentes con un alto grado de conducta antisocial presentaron un consumo mayor de cannabis $[0,12$ vs. $0,79, F(1,904)=104,68, p<0,001]$, derivados morfínicos [0,03 vs. 0,21, $F(1,904)=20,64, p<0,001]$, estimulantes $[0,03$ vs. $0,27, F(1,904)=28,07, p<0,001]$, drogas sintéticas $[0,03$ vs. $0,15, F(1,904)=21,03$, $p<0,001]$ y cocaína $[0,006$ vs. 0,08, $F(1,904)=11,95$, $p<0,01]$, que los grupos caracterizados por una menor presencia de conductas desviadas de las normas de comportamiento social típicas de la edad.

Por su parte, el sexo afectó sólo a la frecuencia de uso de derivados morfínicos $[0,07$ vs. 0,17, F(1,904)= $6,52, p<0,05]$ y estimulantes $[0,05$ vs. $0,25, F(1,904)=$ $18,38, p<0,001$ ], siendo las mujeres las que mostraron 


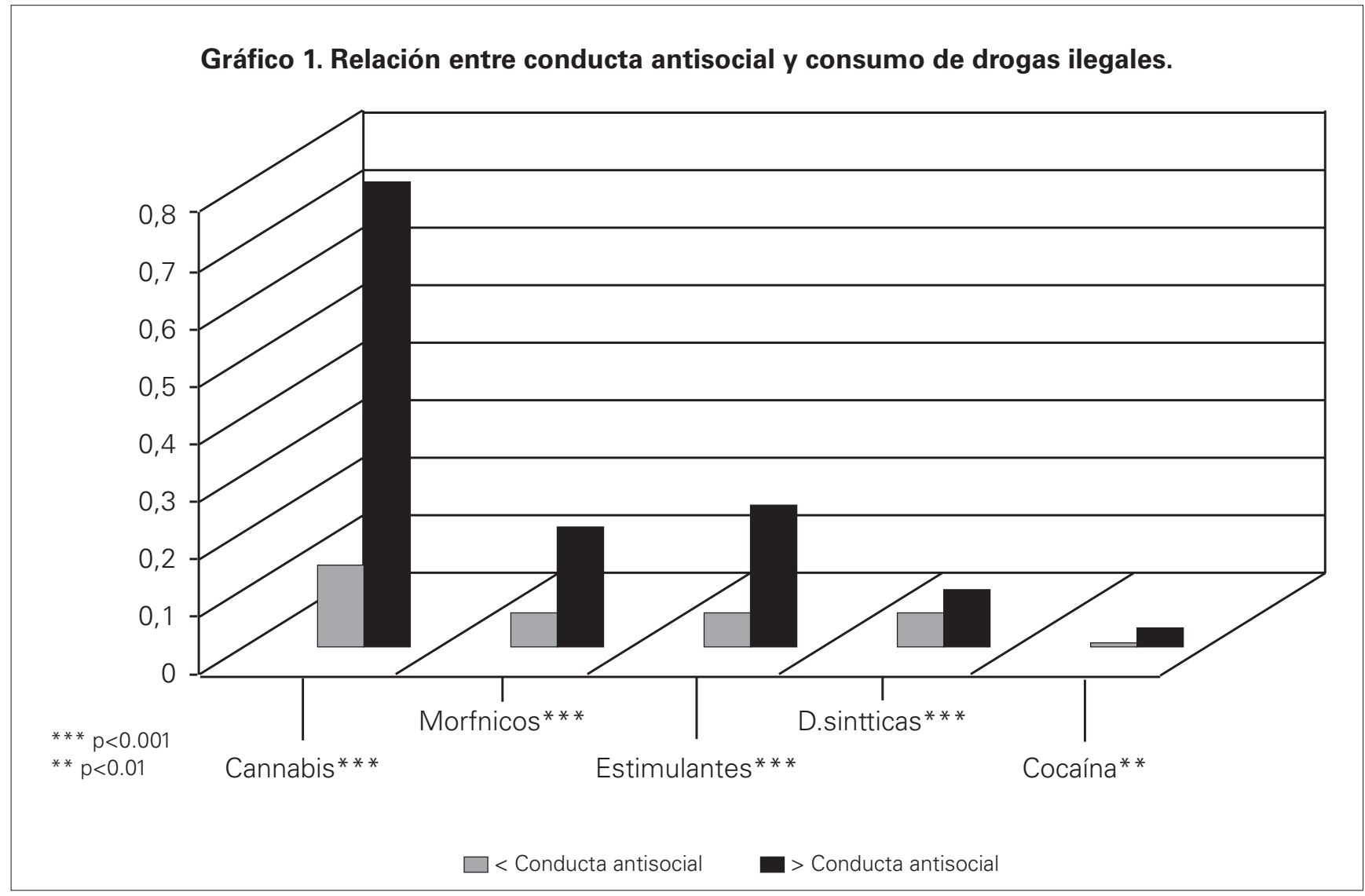

un consumo mayor de ambas sustancias adictivas. Asimismo, en este apartado, se encontró una interacción significativa entre el sexo y el grado de conducta antiso- cial en el caso del uso de estimulantes $[F(1,904)=7,89$, $p<0,01]$ y drogas sintéticas $[F(1,904)=5,61, p<0,05]$, según se presentan en los Gráficos 2 y 3.

\section{Gráfico 2. Interacción conducta antisocial x sexo en el consumo de estimulantes.}

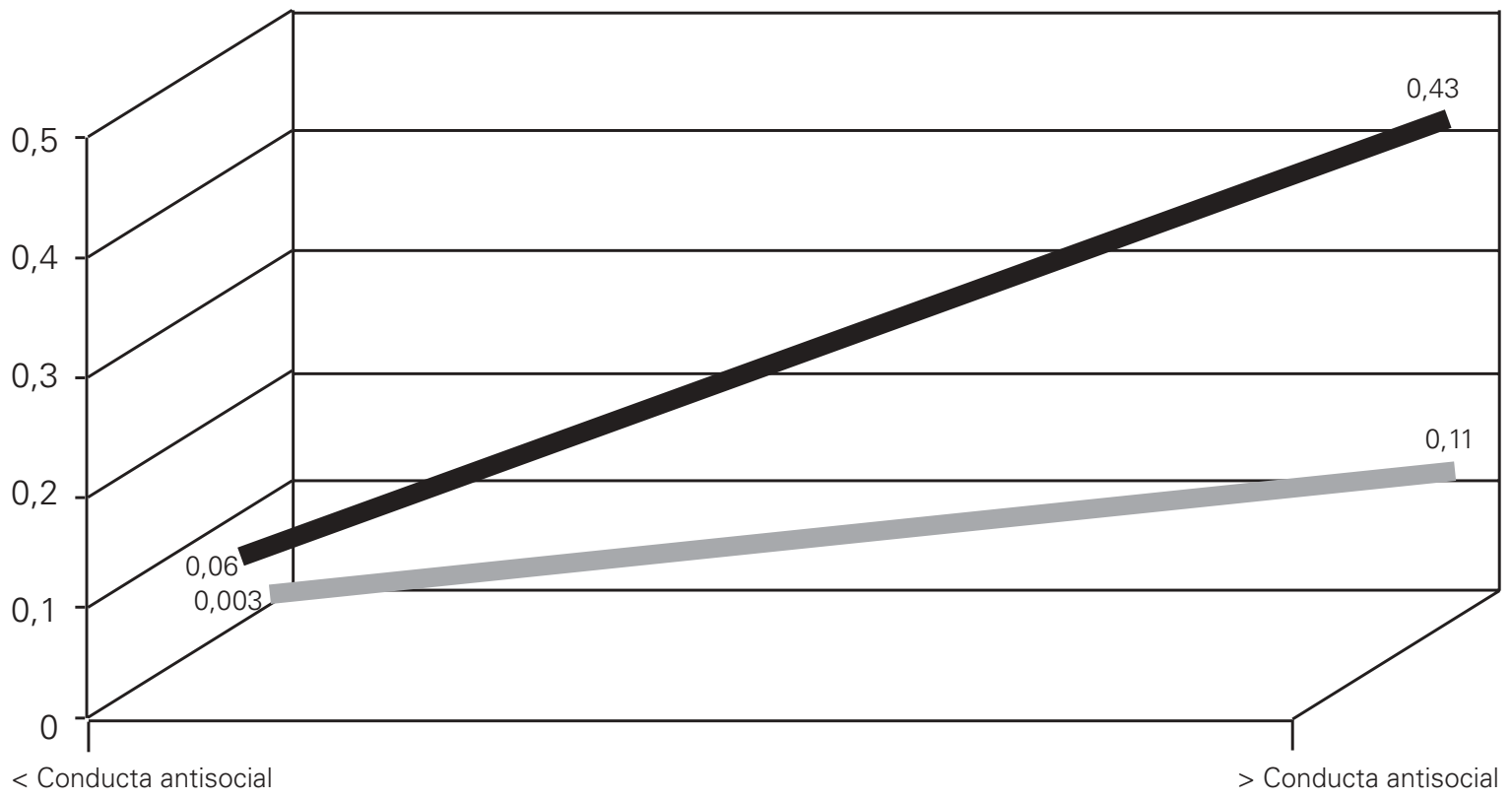




\section{Gráfico 3. Interacción conducta antisocial x sexo en el consumo de drogas sintéticas.}

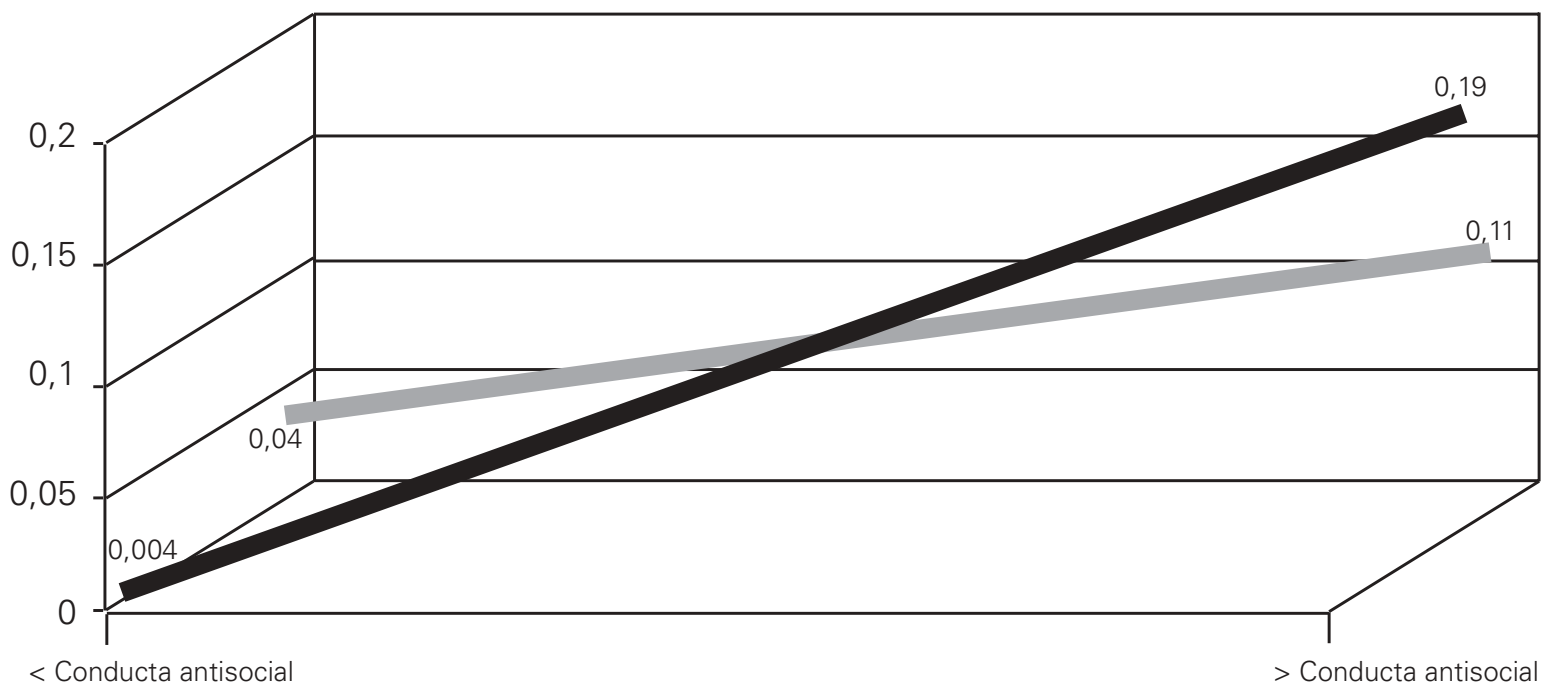

MUJERES

Como se observa, y a diferencia de los varones, las mujeres que exhiben un comportamiento más antisocial realizan un consumo de estimulantes y drogas sintéticas significativamente superior que aquellas que presentan un menor número de conductas desadaptadas.

Respecto al posible influjo de la covariable edad sobre el patrón de consumo de sustancias psicoactivas en función del sexo y de la conducta antisocial de los sujetos, no se observó ningún tipo de influencia de este factor en los respectivos MANOVAS realizados para cada una de las sustancias tenidas en cuenta.

\section{DISCUSIÓN}

Los resultados obtenidos ponen de manifiesto nuevamente la relación existente entre el consumo de drogas y la emisión de conductas desviadas de las normas de comportamiento social tal y como han venido señalando otros estudios tanto de dentro como de fuera de nuestras fronteras (Elliot et al., 1989; Cárdenas y Moreno-Jiménez, 1989; Barnea et al., 1992; Otero López, 1994; Del Barrio y Alonso, 1994; White y Hansell, 1996; White, 1999).

En este sentido, al analizar la relación existente entre la variable conducta antisocial y el tipo de sustan- cia consumida, se ha constatado también la estrecha relación entre un mayor y más frecuente uso de sustancias ilegales y una mayor presencia de conductas contra las normas, confirmando los datos ofrecidos por distintos estudios de diversa índole que subrayan la fuerte relación existente entre el consumo de drogas y la delincuencia, independientemente de los tipos de conducta, tanto de consumo como delictiva, y del tipo de muestra analizada ( Ball y cols., 1982; Johnson et al., 1991; Otero López, 1994; Munñoz-Rivas, 1998).

Así pues, se ha evidenciado que aquellos adolescentes que muestran una frecuencia de consumo mayor de cualquiera de las drogas analizadas (cannabis, derivados morfínicos, cocaína o drogas de síntesis), también obtienen mayores puntuaciones en conducta antisocial.

Al analizar comparativamente por sexos, esta variable se evidencia como un factor modulador relevante, puesto que es la mujer, a diferencia del varón, quien de forma significativa presenta un consumo superior en el caso de los derivados morfínicos y estimulantes. Este hecho confirma los resultados obtenidos por diversos estudios que asocian repetidamente el consumo de estas sustancias al sexo femenino (PNSD, 1994, 1996; Junta de Andalucía, 1997; Muñoz-Rivas, 1998), volviendo a subrayar la preferencia por parte de las mujeres por el uso de fármacos ${ }^{1}$ que posiblemente sea res-

\footnotetext{
En este punto, es necesario aclarar que en el grupo de derivados morfínicos además de la heroína se ha estimado también el uso de otra serie de fármacos con una composición derivada de la codeína o la morfina de posible uso común por su poder analgésico y, también, en el caso de los fármacos estimulantes, entre los que se ha incluido no sólo eluso de anfetaminas sino el de otro tipo de medicamentos comunes que pudieran provocar el mismo efecto farmacológico comoson determinados compuestos de fácil acceso para el tratamiento de insomnio o para el adelgazamiento (por ser más utilizadas por padres y adultos y poderse adquirir a un bajo precio no porque no requieran prescripción médica para su obtención) y, por último, el caso de los tranquilizantes en el que, como en otros estudios, se consideraron todo tipo de psicofármacos de uso común que provocaran efectos similares.
} 
puesta a un consumo menos exteriorizado, más centrado en sustancias de mayor accesibilidad y con menos connotaciones a nivel social (supuestamente, se trata de fármacos que a pesar de exigir receta médica para su comercialización pueden tener una mayor aceptación social al ser indicadas, realizando un buen uso de las mismas, para el tratamiento de dolencias o problemas relacionados con la salud).

Por último, la existencia de un efecto de interacción entre la conducta antisocial y el sexo de los sujetos en el consumo de estimulantes y drogas sintéticas, pone de relieve nuevamente diferencias entre hombres y mujeres puesto que, en el grupo de adolescentes que han obtenido altas puntuaciones en conducta antisocial, son estas últimas las que consumen significativamente estas dos sustancias con mayor frecuencia que los hombres. Por contra, es interesante subrayar que en el grupo de jóvenes con un nivel de conducta antisocial bajo, el consumo de estas sustancias se ve reducido de forma ostensible en ambos sexos, lo que señalaría que aquellos sujetos con una menor frecuencia de consumo sustancias estimulantes y sintéticas llevan a cabo un menor número de conductas desviadas de las normas.

Sin duda, el contenido de los datos expuestos vuelve a confirmar la necesidad de incorporar este tipo de variables en los trabajos dirigidos a la prevención, considerando aquellas propuestas que subrayen, de una forma u otra, alternativas más eficaces para reducir el consumo de sustancias adictivas atendiendo a otras variables como la conducta antisocial. Y, además, adecuar los mismos atendiendo al sexo de los sujetos a las que van dirigidas puesto que, en muchos casos, y como hemos podido comprobar en el presente estudio, esta variable desempeña un papel modulador significativo no sólo en cuanto al grado de implicación del adolescente en el consumo sino también en cuanto al tipo de sustancias de preferencia.

\section{REFERENCIAS BIBILIOGRÁFICAS}

Allsop, J.F. y Feldman, M. (1976). Personality and anti-social behavior in schoolboys: Item analysis of questionnaire measures. British Journal of Criminology, 16, 337-351.

Ball, J.C., Rosen, L., Flueck, J. y Nurco, D. (1982): Lifetime criminality of heroin addicted in the United States. Journal of Drug Issues 12: 225-239.

Barnea, Z., Teichman, M. y Rahav, G. (1992). Personality, cognitive and interpersonal factors in adolescent substance use: A longitudinal test of an integrative model. Journal of Youth and Adolescence, 21, 187-201.

Barnes, G.M. y Welte, J.W. (1986). Patterns and predictors of alcohol use among 7-12th grade students in New York State. Journal of Studies on Alcohol, 47, 53-62.
Brook, Witheman, Gordon y Cohen (1989). Changes in drug involvement: A longitudinal study of childhood and adolescent determinants. Psychological Reports, 65, 707-726.

Brook, J.S., Brook, D.W., Gordon, A.S., Whiteman, M. y Cohen, P. (1990). The psychosocial etiology of adolescent drug use: A family interactional approach. Genetic, Social, and General Psychology Monographs, 116 (2).

Cardenas y Moreno Jiménez (1989). La búsqueda de sensaciones y la ingesta de alcohol en la adolescencia. Revista de Psicología General y Aplicada, 42, 343-349.

Del Barrio y Alonso (1994). Búsqueda de sensaciones y consumo de drogas legales en escolares. Clínica y Salud, 5(1), 69-81.

Elliot, D.S., Huizinga, D. y Menard, S. (1989). Multiple problem youth: Delinquency, substance use and mental health problems. New York: Springer-Verlag.

Gittelman, R.S., Mannuzza, R.S. y Bonagura, N. (1985). Hyperactive boys almost grown up: I. Psychiatric status. Archives of General Psychiatry, 42, 937-947.

Johnson, V. y Pandina, R.J. (1991). Effects of the family envoroment on adolescent substance use, delinquency, and coping styles. American Journal od Drug and Alcohol Abuse, 17, 71-88.

Junta de Andalucía (1997). Plan andaluz sobre drogas. Sevilla: Consejería de Salud. Comisionado para la droga.

Kandel, D.B. (1982). Epidemiological and psychosocial perspectives on adolescent drug use. Journal of American Academic Clinical Psychiatry, 21, 328-347.

Kellam, S.G., Brown, H., Rubin y Ensminger (1983). Paths leading to teenage psychiatric symptoms and substance use: Developmental epidemiological studies in Woodlawn. En S. Guze, F. Earls y J. Barret (Eds.), Childhood psychopathology and development. Nueva York: Raven Press.

Kellam, S.G. y Brown, H. (1982). Social adaptational and psychological antecedents of adolescent psychopathology ten years later. Baltimore: Johns Hopkins University.

Lewis, C.E., Robins, L.N. y Rice, J. (1985). Association of alcoholism with antisocial personality in urban men. Journal of Nervous and Mental Disease, 173, 166174.

Loeber, R. (1988). Natural histories of conduct problems, delinquency, and associated substance use: Evidence for developmental progressions. En B.B. Lahey y A.E. Kazdin (Eds.), Advances in clinical child psychology (Vol. 11). Nueva York: Plenum Press.

Loeber, R.T. y Dishion, T. (1983). Early predictors of male delinquency: A review. Psychological Bulletin, 93, 6899.

McCord, J. (1981). Alcoholism and criminality. Journal of Studies on Alcohol, 42, 739-748.

Muñoz- Rivas, M.J. (1998). Factores de riesgo en drogodependencias: Consumo de drogas en adolescentes. Tesis Doctoral.

Nylander, I. (1979). The development of antisocial behavior in children. Acta Paedopsychiatrica, 47, 71-80. 
Otero-López, J.M. (1994): Droga y delincuencia: Concepto, medida y estado actual del conocimiento. Madrid: Eudema.

PNSD (1994). Encuesta sobre drogas a la población escolar. Madrid: Delegación del Gobierno para el Plan Nacional sobre Drogas.

PNSD (1996). Encuesta sobre drogas a la población escolar (Informe preliminar). Madrid: Delegación del Gobierno para el Plan Nacional sobre Drogas.

Robins, L.N. (1978). Study childhood predictors of adult antisocial behavior: Replications from longitudinal studies. Psychological Medicine, 8, 611-622.

Schukit, M.A. (1983). Extroversion and neuroticism in young men at higher or lower risk for alcoholism. American Journal of Psychiatry, 140, 1223-1224.

Schukit, M.A. (1982). Anxiety and assertiviness in the relatives of alcoholics and controls. Journal of Clinical Psychiatry, 43, 238-239.
Silva, F., Martorell, M.C. y Clemente, A. (1986). Adaptación española de la escala de conducta antisocial ASB: Fiabilidad, validez y tipificación. Evaluación Psicológica, 2(5), 39-55.

Spivack (1983). High risk early behaviors indicating vulnerability to delinquency in the community and school. National Institute of Juvenile Justice and Delinquency Prevention, Office of Juvenile Justice and Delinquency Prevention, Law Enforcement Assistance Administration. Washington DC: U.S. Government Printing Office.

White, J. (1999): The role of alcohol in sexual assault. Aggressive Behavior 25: 1.

White, H. y Hansell, S. (1996): A longitudinal investigation of alcohol use and aggression from adolescence to adulthood. XII ISRA Congress, Louis Pasteur University, Francia. 